\title{
Low-temperature Collisions between Neutral Molecules in Merged Molecular Beams
}

\author{
Benjamin Bertsche§, Justin Jankunas, and Andreas Osterwalder ${ }^{\star}$
}

§SCS-Metrohm Foundation Award for best oral presentation

\begin{abstract}
We have developed an experiment for the investigation of neutral molecular collisions in the gas phase at temperatures as low as $100 \mathrm{mK}$. These low temperatures are obtained by merging two supersonic expansions, using an electric and a magnetic guide, and by matching the velocities of the beams. Since the energy available for the collisions, or the temperature, is determined only by the relative velocity of the reaction partners this enables the study of chemical processes at very low temperatures without the need to prepare slow molecules in the laboratory frame of reference. This paper describes the method and presents results on the $\mathrm{Ne}\left({ }^{3} \mathrm{P}_{2}\right)+\mathrm{NH}_{3}$ Penning ionization.
\end{abstract}

Keywords: Cold chemistry $\cdot$ Merged molecular beams $\cdot$ Molecular scattering $\cdot$ Penning ionization

\section{Introduction}

The study of gas-phase chemical reactions at the highest possible level of control has been the goal of the field of chemical dynamics since its start. Undoubtedly the most important technique in this area for the past half century is the crossed-beam technique. Here, two supersonic expansions are crossed, and reaction products are detected at different angles relative to the incident beams. This technique gave direct access to differential reaction cross sections for the first time, and by controlling the supersonic expansions it was possible to control both the collision energy and the amount of internal energy stored in either of the reactants. These developments were rewarded with the Nobel Prize in $1986,{ }^{[1]}$ and the technique to this day has remained the work horse in experimental reaction dynamics research. Results from

\footnotetext{
${ }^{\star}$ Correspondence: Prof. Dr. A. Osterwalder

EPFL

SB-ISIC

$\mathrm{CH}-1015$ Lausanne

Tel.: +41216937971

E-mail: andreas.osterwalder@epfl.ch
}

such studies represent the basis of our most fundamental understanding of gas phase reactive scattering. A particular class of chemical processes, however, remains elusive to this technique: reactions at low collision energies. Venturing into the regime of lower temperatures is interesting for several reasons: large regions of interstellar space have temperatures of $10 \mathrm{~K}$ or less, and a full understanding of interstellar chemistry requires the knowledge of experimental temperature-dependent cross sections for relevant reactions. ${ }^{[2,3]}$ But low temperatures also represent a fundamental, qualitatively new regime: when the deBroglie wave length $\lambda_{d B}$ of the reaction partners become comparable to the relevant interatomic distances, the quantum mechanical character of a collision process begins to dominate. Most prominent consequences of this are tunneling effects in the reaction, and associated resonances in the reaction cross section. These resonances are not only interesting from a fundamental viewpoint, but they also serve as very sensitive probes for the potential energy surface (PES) on which the reaction takes place. ${ }^{4]}$ Resonances are only one of the many effects that are expected at low temperatures. Extension to low temperatures of concepts like the minimum energy path (MEP) must be thoroughly tested. Alignment of molecules during their approach is another example that may not necessarily apply to low collision energies the same way it does at higher temperatures. Both of these effects can play a role in the outcome of a chemical reaction with multiple channels that lead to different products.
The relative kinetic energy of two particles moving at velocities $v_{i}$ that subtend an angle $\theta$ is given by

$$
E_{\text {coll }} \propto v_{1}^{2}+v_{2}^{2}-2 v_{1} v_{2} \cos (\theta) .
$$

In crossed beam experiments the $v_{i}$ are taken as the velocities of molecular beams, and usually $\theta=\pi / 2$. $\mathrm{E}_{\text {coll }}$ then is the energy available for the reaction, and usually corresponds to temperatures $\mathrm{E}_{\text {coll }} / \mathrm{k}_{\mathrm{b}}>$ $50 \mathrm{~K}\left(\mathrm{k}_{\mathrm{b}}\right.$ being the Boltzmann constant). For the past decades the only method to reach lower temperatures was a method called CRESU (reaction kinetics in uniform supersonic expansions) where one reactant and a precursor to the second one are co-expanded in a cold supersonic expansion. ${ }^{5]}$ The reaction is induced by forming the second reactant in situ, with lasers or electron beams, and measuring the product flux a certain distance downstream. CRESU has reached temperatures as low as $6 \mathrm{~K},{ }^{[6]}$ but achieving lower temperatures is very challenging. Alternative approaches bear on the concept of crossed beams. As seen in Eqn. (1), the collision energy is reduced by reducing either the $v_{i}$ or $\theta$. Control of the velocities is possible nowadays by techniques like Stark- and Zeeman-deceleration, ${ }^{[7]}$ and fundamentally interesting studies have been done using such an approach. ${ }^{[8]}$ However, crossing two molecular beams at right angles poses a fundamental limitation on the lowtemperature limit. Alternatively, the angle between the beams can be reduced while 
keeping the velocities high. Low-angle crossed beam experiments were recently performed and produced temperatures of less than 5 K. ${ }^{[9,10]}$ To reach lower temperatures, the angle needs to be zero, for which a new approach is required: merged molecular beams. This is the method that has been developed in our lab over the past years, and independently in another laboratory, at the Weizmann institute in Israel. ${ }^{[11]}$ Merging beams means overlapping them in space, and setting the relative angle to zero such that the beams move on top of each other, in other words $\theta=0$ and Eqn. (1) becomes $E_{\text {coll }} \propto\left(v_{1}-v_{2}\right)^{2}$ and, in particular, $E_{\text {coll }}=0$ when $v_{1}=v_{2}$. But the merging of two beams cannot be achieved without bending at least one of the beams onto the axis of the second beam, and this requires technologies that were developed in the past decade mainly in the field of cold molecules: bent electric or magnetic guides for neutral molecules.

With these technologies at hand it is now possible to study many reactions between small polar and paramagnetic molecules over a temperature range spanning more than three decades, from few $\mathrm{mK}$ up to almost room temperature. Several studies on fundamental electron transfer processes, called Penning Ionization (PI), have already been performed and point the way in which this field will progress in the next years.[11-13] While until now mostly atoms and diatomic molecules were investigated, ${ }^{[11]}$ the richness of low-temperature chemistry becomes apparent when studying polyatomic molecules. Here we can, for example, study the branching ratio for the different reaction channels and relate them to the shape of the PES and the dynamics of the collision process thereon.

\section{Experimental}

The experimental setup is shown in Fig. 1. Two pulsed skimmed supersonic expansions, labeled as SE1 and SE2 in the figure, produce molecular beams of paramagnetic and polar particles that are directed into the magnetic (MG) and electric guides (EG), respectively. In the present experiment, SE1 produces excited neon atoms (designated as $\mathrm{Ne}^{*}$ ), while $\mathrm{SE} 2$ produces a beam of $\mathrm{NH}_{3}$ molecules. $\mathrm{Ne}^{*}$ is generated by crossing a pure $\mathrm{Ne}$ expansion with fast electrons. The speeds of the beams, adjusted via the choice of carrier gas and temperature, can be varied between $500 \mathrm{~m} / \mathrm{s}$ and $1100 \mathrm{~m} / \mathrm{s}$. The slowest speeds are reached by cooling the pulsed valves to $170 \mathrm{~K}$ using liquid nitrogen. A continuously tunable velocity is achieved by heating the cooled valve to any desired temperature up to $320 \mathrm{~K}$.

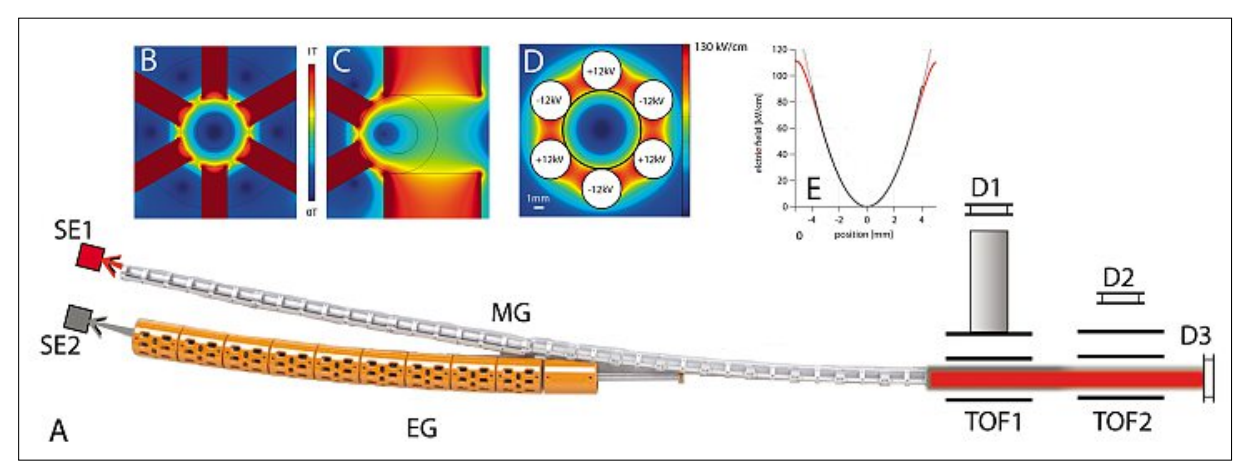

Fig. 1. Experimental setup. Panel A shows the overview of the setup, as explained in the text. Panels $\mathrm{B}$ and $\mathrm{C}$ show cross sections through the magnetic field distributions in the first and second part of the magnetic guide, respectively. Panels $D$ and $E$ are two- and one-dimensional cross sections through the electric field distribution in the electrostatic guide.

\section{The Guides}

The beam of paramagnetic particles is bent in a magnetic guide (MG in Fig. 1A). This guide is constructed from NdFeB permanent magnets with remanent magnetic fields of $1.17 \mathrm{~T}$. The first section of the guide, shown in cross-section in Fig. 1B, is straight with an inner opening diameter of $8 \mathrm{~mm}$, has hexapole symmetry, and a potential depth of about $0.7 \mathrm{~T}$. The second section, Fig. 1C, is an open quadrupole arrangement that leaves enough space for the beam of polar molecules to be transmitted and overlapped with the paramagnetic beam. The end of the magnetic guide is parallel to the end of the electrostatic guide to ensure perfect merging, and the interaction region (inside TOF1 in Fig. 1) is 30 $\mathrm{cm}$ downstream of the end of the magnetic guide.

The electric guide (EG) is constructed from six cylindrical electrodes positioned on the corners of a regular hexagon with an inner opening of $8 \mathrm{~mm}$ diameter. Application of alternating positive and negative voltages of equal magnitude produces a hexapole field inside the guide, with a minimum at the center, as shown in Fig. 1E. In order to bend the molecular beam we have constructed the guide from ten straight segments, each $10 \mathrm{~cm}$ long that are connected with an angle of one degree at the joints. The resulting guide has a bend radius of almost 6 meters and performs a curve of 10 degrees, which is sufficient to remove the end of the guide from the line of sight of the expansion. This ensures that no molecule or atom with an unsuitable Stark effect reaches the end of the guide. The beam emerging from the electrostatic guide is recollimated by an electrostatic hexapole lens at the end of the guide. This lens focuses the beam of polar molecules through the magnetic guide into the collision region.

\section{The Interaction Region}

The two beams are merged at the end of the magnetic guide. The reaction starts as soon as the two beams overlap, but the detection of reaction products only happens $30 \mathrm{~cm}$ behind the end of the magnetic guide. In the $\mathrm{Ne}^{*}+\mathrm{NH}_{3}$ PI reaction experiments the products were detected in a timeof-flight mass spectrometer (TOF-MS) installed perpendicularly to the beam propagation axes (TOF1 in Fig. 1A). The extraction voltage on the TOF-MS is switched on for a few $100 \mathrm{~ns}$ at a specific time after the firing of the supersonic expansions in order to precisely select a small section of the initial velocity distribution. Given by the duration of the molecular pulse and the velocity spreads in the beams, we obtain a velocity spread of about $30 \mathrm{~m} / \mathrm{s}$ for the current reaction system. The combination of the beam divergence during the travel from the valve to the interaction region with the short extraction times reduces the velocity spread considerably below the initial width of the velocity distribution. Further reduction would be possible by attaining higher speed ratios, shorter molecular pulses, or shorter extraction times. ${ }^{[14]}$

\section{Beam Monitoring}

Since the pulsed valves produce molecular number densities that vary with different expansion conditions, and in some cases also from pulse to pulse, it is critical to continuously monitor the density in both beams during the collection of reaction products. This is achieved by using two detectors installed $50 \mathrm{~cm}$ behind the interaction region (TOF2 and D3 in Fig. 1A). In the present case of a $\mathrm{Ne}^{*}$ and an $\mathrm{NH}_{3}$ beam the $\mathrm{Ne}^{*}$ density is measured by directly impinging the atomic beam onto a microchannel plate detector (D3). $\mathrm{NH}_{3}$ molecules are state-selectively photoionized, and the resulting ions are measured, together with the laser power, at every molecular pulse, using D2.

During an experiment for the $\mathrm{Ne}^{*}+$ $\mathrm{NH}_{3}$ reaction the velocity of the $\mathrm{Ne}^{*}$ beam is set by controlling the temperature of the pulsed valve for a pure $\mathrm{Ne}$ expansion. Velocities in the range of $500-800 \mathrm{~m} / \mathrm{s}$ are 
obtained in this manner. $\mathrm{NH}_{3}$ is expanded either pure or seeded in argon to obtain beam velocities of $1090 \mathrm{~m} / \mathrm{s}$ and $610 \mathrm{~m} / \mathrm{s}$, respectively. The lens voltage is adjusted for optimum beam overlap with the $\mathrm{Ne}^{*}$ beam. The relative timing of the two valves is adjusted such that the desired velocity components from both expansions reach the interaction region at the same time, and the switching of the extraction voltages in the TOF-MS is synchronized with those velocities. The laser for beam density monitoring is timed to measure only the density of the particular velocity segment that participated in the observed reactions, and the amplitude of the $\mathrm{Ne}^{*}$ signal is read out at that particular time only. Reaction rates are obtained by counting product ions from PI, and converted to rate constants by normalizing with the measured beam densities. Cross sections are then given by the rate constant divided by the relative velocity.

\section{Results}

The example presented here is the PI of $\mathrm{NH}_{3}$ molecules in collisions with $\mathrm{Ne}^{*}$. The energy level diagram shown in Fig. 2 reveals the channels through which this reaction can proceed. Of the three spin-orbit components of the lowest $\mathrm{Ne}^{3} \mathrm{P}$ state only the $\mathrm{J}=2$ reaches the end of the guide. $\mathrm{J}=0$ is not paramagnetic and $\mathrm{J}=1$ is short-lived. $\mathrm{Ne}\left({ }^{3} \mathrm{P}_{2}\right)$ has an internal energy of $16.7 \mathrm{eV}$. Since the ionization potential of $\mathrm{NH}_{3}$ is only $10.2 \mathrm{eV}$ the ammonia molecule can transfer an electron to the neon atom which then ejects the electron from the higher orbital. This way a reactive collision forms $\mathrm{NH}_{3}^{+}$cations, neutral ground state $\mathrm{Ne}$, and a free electron. The two electronic states of $\mathrm{NH}_{3}^{+}$shown in Fig. 2 correspond to removal of an electron from two different orbitals of $\mathrm{NH}_{3}$ : from the lone pair, leading to ground state $\mathrm{NH}_{3}{ }^{+}$, or from one of the N-H bonding orbitals, leading to the excited $\mathrm{A}$ state. This state is produced with sufficient internal energy to dissociate into $\mathrm{NH}_{2}^{+}+\mathrm{H}$ products. These processes have been observed in previous studies at collision energies above $50 \mathrm{meV}$ (approximately $300 \mathrm{~K}$ ), where the channels were distinguished by the kinetic energy of the ejected electron. ${ }^{[15]}$ In our experiment we can distinguish the two reaction channels by mass-selective detection of the reaction products, and we can thus measure the reaction cross sections and branching ratio over the entire temperature range available in our experiment. Fig. 3A shows the rate constant for direct $\mathrm{NH}_{3}{ }^{+}$production, and Fig. 3B the branching ratio for $\mathrm{NH}_{2}^{+}$production for the accessible collision energies, corresponding to temperatures between $100 \mathrm{mK}$ and $250 \mathrm{~K}$. The rate constant increases with increasing temperature, as is expected for a process that is determined by long-range interactions. Indeed, a fit of the lowest six data points to a function $\log (k)=x \log \left(E_{\text {coll }}\right)$ produces $x=0.23$ as shown by the red line in Fig. 3A. This parameter is related to the shape of the interaction potential by $V(r)=a r^{\frac{2}{x-1 / 2}}[16]$ (where $r$ is the distance between the reactants), and the value of $x$ found here corresponds to a long-range interaction potential similar to $V(r)=\frac{a}{r^{6}}$. At higher temperatures the rate constant deviates from this curve, which indicates the influence of other terms in the interaction potential that describe short-range interactions. The truly surprising observation is the branching ratio, which is constant over the entire range of collision energies studied here. Extending the MEP concept, which has proven very successful at high temperatures, to low temperatures may lead to the assumption that at the lowest collision energies the only reaction product should be $\mathrm{NH}_{3}^{+}$, because the most stable geometry of the $\mathrm{Ne}^{*}-\mathrm{NH}_{3}$ complex shows the $\mathrm{Ne}^{*}$ binding through the $\mathrm{NH}_{3}$ lone pair. We can speculate on the reason for the observation of a temperature-independent branching ratio, but for a complete understanding extensive calculations will be required that at this point cannot be performed. In the temperature range studied here $\lambda_{d B}$ of $\mathrm{Ne}$ and $\mathrm{NH}_{3}$ decreases from $\approx 20 \AA$ to subatomic dimensions. One may expect a concept like the MEP to fail if $\lambda_{d B}$ becomes larger than the width of the required potential well on the PES.

While we do not yet have a complete understanding of the observed processes, and complete theoretical calculations are not yet possible, our results give a glimpse

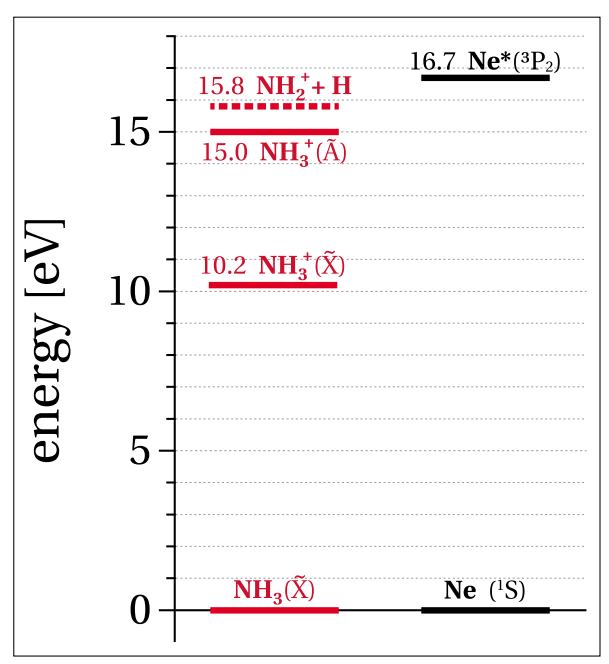

Fig. 2. Energy level diagram showing the relevant states of $\mathrm{NH}_{3}$ and $\mathrm{Ne}$.

of what will be possible in low-temperature chemistry in the near future. Scattering resonances, the main effect sought in the past few years are a very important aspect that will be very useful for a quantitative characterization of PES for chemical reactions. However, as the colliding partners become heavy the resolution of such resonances becomes ever more difficult. So far, resonances have been observed only in collision systems with a reduced mass below 10 amu. ${ }^{[9-11]}$ The present work demonstrates that in going to more complex systems quite different effects become accessible. Complex systems normally offer multiple reaction paths, and the temperature-dependent branching ratios may be very different at low temperatures than at high ones. One even has to ask, in this context, where the

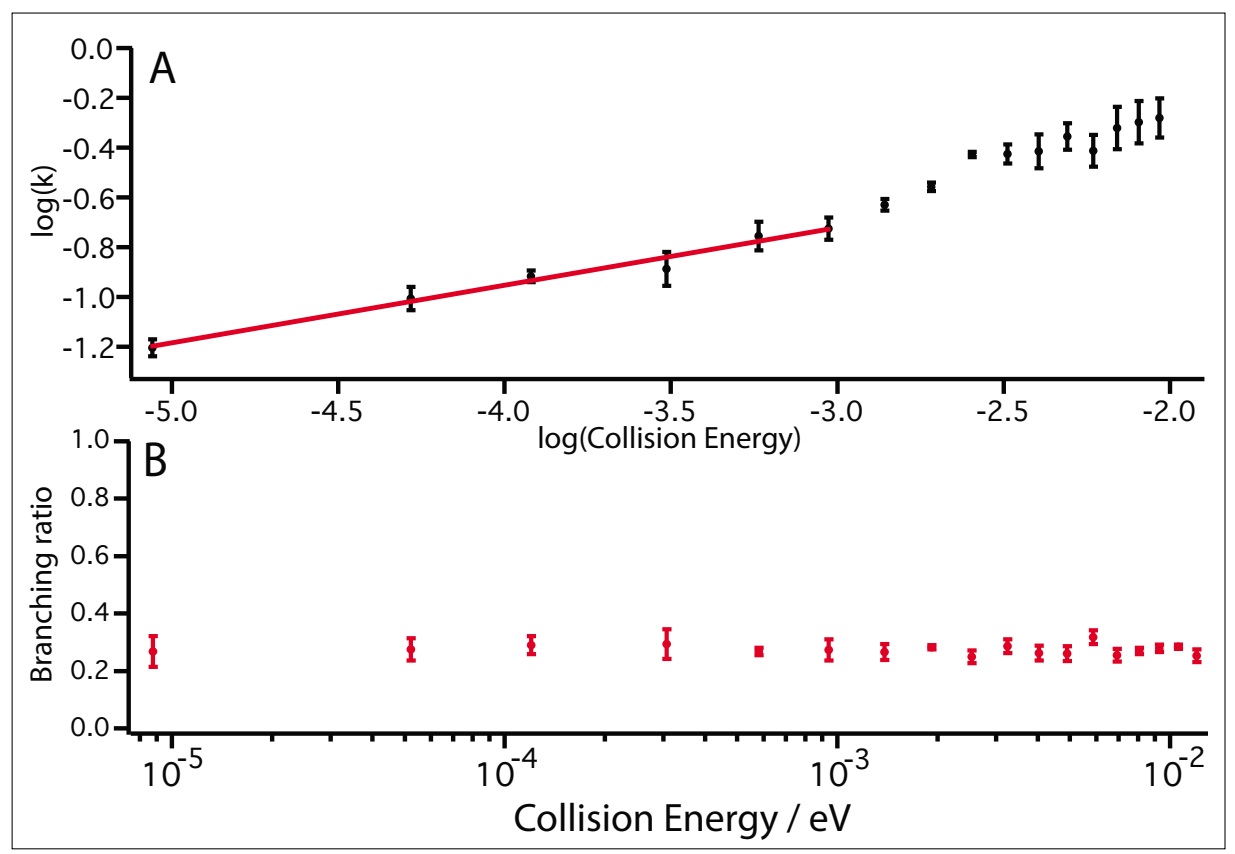

Fig. 3. A: Measured reaction rate constants (black symbols) and fit of the lowest six data points (red line, see text). B: Branching ratio for $\mathrm{NH}_{2}^{+}$vs. $\mathrm{NH}_{3}^{+}$production for $\mathrm{Ne}^{*}+\mathrm{NH}_{3}$ Penning ionization. 
low-temperature regime starts and how this depends on the observed system. As in the case of the $\mathrm{Ne}\left({ }^{3} \mathrm{P}_{2}\right)+\mathrm{NH}_{3}$ reaction shown here, the branching ratios often depend on the angle of incidence of the reactants. It will be interesting to combine low-temperature collision studies with alignment of the reactants prior to the reaction. The MEP concept postulates an adiabatic orbital following, and thus an alignment of $\mathrm{NH}_{3}$ into the most stable $\mathrm{Ne}\left({ }^{3} \mathrm{P}_{2}\right)-\mathrm{NH}_{3}$ structure, as the collision energy vanishes. Extrinsic alignment of the molecule using, e.g. lasers may then lead to different branching ratios and can be used to further explain the experimental observations. Taking these concepts even further will be the application to barrierless chemical reactions between radicals and polar molecules. These are interesting not only from a fundamental viewpoint but in particular also for interstellar applications.

The study of chemical dynamics below $10 \mathrm{~K}$ has been sought after for many decades and only now has it become pos- sible. We expect many fundamentally new insights into the very basics of chemistry, and look forward to moving into new and unknown territories!

\section{Acknowledgments}

We thank the mechanical workshop at ISIC for their enthusiasm and support in the construction of this experiment. This work is funded by the Swiss Science Foundation (Project Number PP0022-119081) and the EPFL.

Received: January 12, 2014

[1] http://www.nobelprize.org/nobel_prizes/ chemistry/laureates/1986.

[2] W. Klemperer, Annu. Rev. Phys. Chem. 2011, $62,173$.

[3] I. W. M. Smith, Annu. Rev. Astro. Astrophys. 2011, 49, 29.

[4] F. Fernández-Alonso, R. N. Zare, Annu. Rev Phys. Chem. 2002, 53, 67.

[5] I. R. Sims, I. W. M. Smith, Annu. Rev. Phys. Chem. 1995, 46, 109

[6] C. Berteloite, M. Lara, A. Bergeat, S. D. Le Picard, F. Dayou, K. M. Hickson, A. Canosa, C. Naulin, J.-M. Launay, I. R. Sims, Phys. Rev Lett. 2010, 105, 203201.
[7] S. Y. T. van de Meerakker, H. L. Bethlem, N. Vanhaecke, G. Meijer, Chem. Rev. 2012, 112, 4828.

[8] S. Y. T. van de Meerakker, G. Meijer, Faraday Discuss. 2009, 142, 113

[9] S. Chefdeville, T. Stoecklin, A. Bergeat, K. M. Hickson, C. Naulin, M. Costes, Phys. Rev. Lett. 2012, 109, 23201.

[10] S. Chefdeville, Y. Kalugina, S. Y. T. van de Meerakker, C. Naulin, F. Lique, M. Costes, Science 2013, 341, 1094

[11] A. B. Henson, S. Gersten, Y. Shagam, J. Narevicius, E. Narevicius, Science 2012, 338, 234.

[12] B. Bertsche, K. Jachymski, J. Jankunas, M. Hapka, Z. Idziaszek, A. Osterwalder, submitted.

[13] E. Lavert-Ofir, Y. Shagam, A. B. Henson, S. Gersten, J. Kłos, P. S. Zuchowski, J. Narevicius, E. Narevicius, Nature Chem. 2014, doi:10.1038/ nchem. 1857.

[14] Y. Shagam, E. Narevicius, J. Phys. Chem. C 2013, 117, 22454.

[15] M. Ben Arfa, B. Lescop, M. Cherid, B. Brunetti, P. Candori, D. Malfatti, S. Falcinelli, F. Vecchiocattivi, Chem. Phys. Lett. 1999, 308, 71.

[16] A. Niehaus in 'Adv. Chem. Phys. XLV', Ed. J. W. McGowan, JohnWiley \& Sons, 1981, p. 399. 